\title{
On s-extremal Riemann surfaces of even genus
}

\author{
Ewa Kozłowska-Walania ${ }^{1}$
}

Received: 23 February 2020 / Accepted: 6 November 2020 / Published online: 24 November 2020

(c) The Author(s) 2020

\begin{abstract}
We consider Riemann surfaces of even genus $g$ with the action of the group $\mathcal{D}_{n} \times \mathbb{Z}_{2}$, with $n$ even. These surfaces have the maximal number of 4 non-conjugate symmetries and shall be called s-extremal. We show various results for such surfaces, concerning the total number of ovals, topological types of symmetries, hyperellipticity degree and the minimal genus problem. If in addition an $s$-extremal Riemann surface has the maximal total number of ovals, then it shall simply be called extremal. In the main result of the paper we find all the families of extremal Riemann surfaces of even genera, depending on if one of the symmetries is fixed-point free or not.
\end{abstract}

Keywords Riemann surface - Symmetry of a Riemann surface - Real form . Automorphisms of Riemann surface · Fuchsian groups · Riemann uniformization theorem $\cdot$ Separating symmetry

Mathematics Subject Classification Primary 30F99 - 14H37; Secondary 20F

\section{Introduction}

All Riemann surfaces in this paper are compact. A symmetry of a Riemann surface $X=$ $\mathcal{H} / \Gamma$ of genus $g \geq 2$, where $\Gamma$ is a Fuchsian surface group, is just an antiholomorphic involution $\sigma \in G=\operatorname{Aut}^{ \pm}(X)$. The set of points fixed by $\sigma$ consists of no more than $g+1$ disjoint simple closed curves called ovals, see Harnack [10]. If the set $X \backslash \operatorname{Fix}(\sigma)$ is disconnected, then we call $\sigma$ to be separating and we call it non-separating in the other case. In addition, we define the topological type of $\sigma$ to be a symbol $\pm k$, where

Supported by Polish National Sciences Center by the Grant NCN MINIATURA 3 DEC-2019/03/X/ST1/01239.

Ewa Kozłowska-Walania retrakt@mat.ug.edu.pl

1 Institute of Mathematics, Faculty of Mathematics, Physics and Informatics, University of Gdańsk, Wita Stwosza 57, 80-952 Gdańsk, Poland 
$k \geq 0$ denotes the number of ovals of $\sigma$, and the sign depends on the separability of $\sigma:+$ for separating, - for a non-separating symmetry.

The starting point for this paper is the results of Gromadzki and Izquierdo from $[6,7]$, where they prove that the number of non-isomorphic Klein surfaces $(X, \sigma)$ for a fixed compact Riemann surface $X$ of even genus is at most 4, and this bound is attained only for the automorphism group of the underlying surface being isomorphic to $\mathcal{D}_{n} \times \mathbb{Z}_{2}$, where $\mathcal{D}_{n}$ denotes the dihedral group of order $2 n$ and $\mathbb{Z}_{2}$ is the group of order 2 , for some even integer $n$. Also, the bound for the total number of ovals of the set of symmetries, in terms of $g$ and $n$ is proposed. By an extremal Riemann surface of even genus we shall understand a surface which attains the maximal number of 4 non-conjugate symmetries and the symmetries have the maximal possible total number of ovals- the bound is given in terms of $g$ and $n$. If the surface of even genus has 4 conjugacy classes of symmetries, without any assumption on the number of ovals, then it shall be called s-extremal. In general, a Riemann surface shall be called $s$-extremal if it admits the maximal number of non-conjugate symmetries, o-extremal if it admits the maximal total number of ovals for a configuration of $k$ non-conjugate symmetries, and simply extremal if both conditions hold simultaneously.

The case of commuting symmetries, that is $n=2$, was considered in the author's previous paper [12], where all possible topological types of the 4 symmetries were found. In this paper we shall mainly focus on the non-commuting case, that is $n \geq 4$. The goal of the first part of the paper is to find all possible topological types of symmetries of extremal Riemann surfaces of even genera in the non-commuting case. We consider two cases, letting one of the symmetries be fixed point free or not. As a result of these investigations we obtain a family of Riemann surfaces, which from now on shall be called the button-like Riemann surfaces. These are the only extremal Riemann surfaces of even genera with a non-abeliam automorphism group and one fixed point free symmetry. An interesting property of these surfaces is that one of the central symmetries is fixed point free whereas the other has the maximum of $g+1$ ovals. We also investigate the hyperellipticity degree of such a surface. In this part of the paper we show various results on s-extremal Riemann surfaces of even genera. For example we improve the bound on the total number of ovals given in [7]. We also show that a Riemann surface of even genus has at most 3 separating symmetries. For completeness, we also investigate the case when all the symmetries have fixed points. This one, however, is not so interesting, as it only allows one symmetry type configuration for any even $g$.

In the next part of the paper we find the minimal genus for an $s$-extremal Riemann surface whose automorphism group is isomorphic to $\mathcal{D}_{n} \times \mathbb{Z}_{2}$.

The next part of the paper focuses on the problem of the minimal possible number of ovals. In [9] we provided some results concerning the minimal number of ovals that an $s$-extremal Riemann surface can admit. Here we provide a similar result in the case of even genus, by allowing one of the symmetries to be fixed-point free.

The last part of the paper stands in opposition to the title, as it concerns Riemann surfaces of odd genus, but there is a common trait which justifies it. Namely, of all the $o$-extremal Riemann surfaces, only the ones with 4 or 5 symmetries can be realized with a non-abelian group. As the methods are exactly the same, we provide 
the topological types of 5 symmetries on an $o$-extremal Riemann surface with nonabelian automorphism group. Note, that the abelian case was solved in [13].

\section{Preliminaries}

\subsection{Non-euclidean crystallographic groups}

We shall use the combinatorial approach, based on Riemann uniformization theorem, Fuchsian groups and non-euclidean crystallographic groups. Recall that an NEC group is just a discrete and cocompact subgroup of the group $\mathcal{G}$ of isometries of the hyperbolic plane $\mathcal{H}$, including those which reverse orientation, and if such a subgroup contains only orientation preserving isometries, then it is called a Fuchsian group. For every NEC group $\Lambda$ we have the associated signature, which determines its algebraic structure. It has the form

$$
\left(h ; \pm ;\left[m_{1}, \ldots, m_{r}\right] ;\left\{C_{1}, \ldots, C_{k},(-)^{l}\right\}\right) .
$$

The numbers $m_{i} \geq 2$ are called the proper periods, the non-empty brackets $C_{i}=$ $\left(n_{i 1}, \ldots, n_{i s_{i}}\right), i=1, \ldots, k$, or the empty ones $(-)$ are the period cycles, the numbers $n_{i j} \geq 2$ are the link periods and $h \geq 0$ is said to be the orbit genus of $\Lambda$. The orbit space $\mathcal{H} / \Lambda$ is a surface of topological genus $h$, having $k$ boundary components, and being orientable or not according to the sign being + or - .

The group with the signature (1) has the presentation given by the following generators and relations where $s_{i}=0$ if $i>k$ :

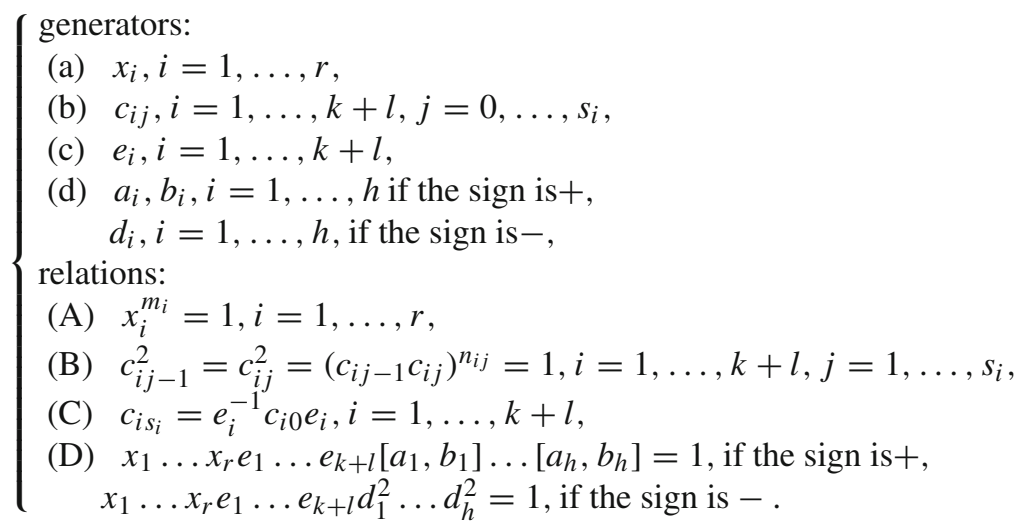

The generators $x_{1}, \ldots, x_{r}$ are called canonical elliptic generators, $e_{1}, \ldots, e_{k+l}$ are called the canonical connecting generators and $c_{i j}$ are the canonical reflections of $\Lambda$.

A Fuchsian group can be regarded as an NEC group with the signature

$$
\left(h ;+;\left[m_{1}, \ldots, m_{r}\right] ;\{-\}\right)
$$


which is usually shortened to $\left(h ; m_{1}, \ldots, m_{r}\right)$; a Fuchsian group without proper periods is called a Fuchsian surface group. An epimorphism $\theta: \Lambda \rightarrow G$, where $\Lambda$ is an NEC group and $G$ is a finite group, is said to be smooth if $\operatorname{ker} \theta$ is a surface group.

Any set of generators of an NEC group satisfying the above relations is called $a$ canonical set of generators, and reflections $c_{i j-1}, c_{i j}$ are said to be consecutive. Every NEC group has an associated fundamental region, whose hyperbolic area $\mu(\Lambda)$, for an NEC group $\Lambda$ with signature (1), is given by

$$
2 \pi\left(\varepsilon h+k+l-2+\sum_{i=1}^{r}\left(1-\frac{1}{m_{i}}\right)+\frac{1}{2} \sum_{i=1}^{k+l} \sum_{j=1}^{s_{i}}\left(1-\frac{1}{n_{i j}}\right)\right)
$$

where $\varepsilon=2$ if the sign is + and $\varepsilon=1$ otherwise.

Now, if $\Gamma$ is a finite index subgroup in an NEC group $\Lambda$, then it is an NEC group itself and the Hurwitz-Riemann formula applied to the covering $\mathcal{H} / \Gamma \rightarrow \mathcal{H} / \Lambda$ says

$$
[\Lambda: \Gamma]=\frac{\mu(\Gamma)}{\mu(\Lambda)} .
$$

Finally it is known that an abstract group with the presentation given by the generators and the relations in (2) can be realized as an NEC group $\Lambda$ with the signature (1) if and only if (4) is positive.

Let us also remind, that the notion of maximality is explained in [4]. It is used in this paper to ensure, that the surfaces obtained here have the required total automorphism groups.

\subsection{Topological type of a symmetry}

To retrieve the topological type of a symmetry, one needs to find its number of ovals and determine its separability type. The following two results are essential in solving this problem.

Theorem 2.1 (Gromadzki [5]) Let $X=\mathcal{H} / \Gamma$ be a Riemann surface whose group of automorphisms is $G=\Lambda / \Gamma$ for some NEC group $\Lambda$ containing $\Gamma$ as a normal subgroup, let $G=\Lambda / \Gamma$ for some NEC group $\Lambda$ and let $\theta: \Lambda \rightarrow G$ be the canonical epimorphism. Then the number of ovals of a symmetry $\sigma$ of $X$ equals

$$
\sum\left[C\left(G, \theta\left(c_{i}\right)\right): \theta\left(C\left(\Lambda, c_{i}\right)\right)\right],
$$

where $C$ stands for the centralizer and the sum is taken over a set of representatives of all conjugacy classes of canonical reflections whose images under $\theta$ are conjugate to $\sigma$.

For a symmetry $\sigma$ we shall denote by $\|\sigma\|$ the number of its ovals. In addition, we denote $\|X\|$ the number of ovals of all non-conjugate symmetries on the surface $X$.

The centralizers that appear in Theorem 2.1 have been described by Singerman in [15] and we shall use the following result, where $*$ stands for free product. 
Theorem 2.2 (Singerman [15]) Let $c_{0}, c_{1}, \ldots, c_{s}$, e be the system of canonical reflections corresponding to a period cycle $\left(n_{1}, \ldots, n_{s}\right)$ of an NEC group $\Lambda$ with signature (1). If all $n_{i}$ are even, then the centralizer $C\left(\Lambda, c_{i}\right)$ equals

$$
\begin{array}{ll}
\left\langle c_{i}\right\rangle \times\left(\left\langle\left(c_{i-1} c_{i}\right)^{n_{i} / 2}\right\rangle *\left\langle\left(c_{i} c_{i+1}\right)^{n_{i+1} / 2}\right\rangle\right)=\mathbb{Z}_{2} \times\left(\mathbb{Z}_{2} * \mathbb{Z}_{2}\right) & \text { for } i \neq 0, \\
\left\langle c_{0}\right\rangle \times\left(\left\langle\left(c_{0} c_{1}\right)^{n_{1} / 2}\right\rangle *\left\langle e^{-1}\left(c_{s-1} c_{s}\right)^{n_{s} / 2} e\right\rangle\right)=\mathbb{Z}_{2} \times\left(\mathbb{Z}_{2} * \mathbb{Z}_{2}\right) & \text { for } i=0, \\
\left\langle c_{0}\right\rangle \times\langle e\rangle=\mathbb{Z}_{2} \times \mathbb{Z} & \text { for } s=0 .
\end{array}
$$

Now to determine the separability type, one can use the following technique described by Hoare and Singerman in [11]. Let us recall, that if $\Phi$ is a set of generators for $\Lambda$ and $\tilde{\Lambda}$ is a subgroup of $\Lambda$, then a right Schreier transversal $S$ is a set of words in $\Phi$ such that every initial segment of a word in $S$ is also in $S$ and the mapping $a \rightarrow \tilde{\Lambda} a$ is a $1-1$ correspondence from $S$ to the cosets of $\tilde{\Lambda}$ in $\Lambda$. Now for each $a \in S$ and $\alpha \in \Phi$ there exists a unique $b \in S$ such that $\tilde{\Lambda} b=\tilde{\Lambda} a \alpha$. With these notations, the Schreier generators of $\tilde{\Lambda}$ are all the elements of the form $a \alpha b^{-1}$.

If $\Lambda$ is a group with generators $\Phi$ and $\tilde{\Lambda}$ is its subgroup, then the Schreier coset graph $\mathcal{K}$ is the graph whose vertices are the cosets of $\tilde{\Lambda}$ in $\Lambda$ and labelled directed edges at every vertex for each $a \in \Phi$ such that $a: \tilde{\Lambda} \alpha \rightarrow \tilde{\Lambda} \alpha a$. Now if $a$ is a reflection and $\tilde{\Lambda} \alpha a=\tilde{\Lambda} a$, then the corresponding directed edge $a: \tilde{\Lambda} \alpha \rightarrow \tilde{\Lambda} \alpha a$ is called a reflection loop. Let us delete all the reflection loops from $\mathcal{K}$. In such a way we obtain the Schreier graph $\overline{\mathcal{K}}$. Observe, that each path in $\overline{\mathcal{K}}$ corresponds to a word in $\Phi$, and so also to an element of $\Lambda$. We shall call a path positive if it corresponds to an orientation preserving element of $\Lambda$, otherwise we shall call a path negative.

We are ready to state the main result of [11]:

Theorem 2.3 (Hoare and Singerman [11]) With the above notations, the following statements are equivalent:

(i) $X / \tilde{\Lambda}$ is orientable

(ii) the only orientation reversing Schreier generators are involutions (actually conjugations in $\Phi)$

(iii) all circuits of $\overline{\mathcal{K}}$ are positive

(iv) the cosets of $\tilde{\Lambda}$ in $\Lambda$ divide into two disjoint classes such that in the action $a$ : $\Lambda \gamma \mapsto \Lambda(\gamma a)$ orientation preserving elements of $\Phi$ fix the classes and (apart from reflections fixing cosets) orientation reversing elements interchange the classes.

Now let us assume that we have an epimorphism $\theta: \Lambda \rightarrow G$, where $\Lambda$ is an NEC group and $G \cong \Lambda / \Gamma$ for $\Gamma=\operatorname{ker} \theta$ is the group of all, including orientation reversing, automorphisms of $X=\mathcal{H} / \Gamma$. Let us also assume that $\sigma \in G$ is a symmetry of $X$. Now let us consider the subgroup $\Gamma_{\sigma}=\theta^{-1}(\langle\sigma\rangle)$ of $\Lambda$. It is an NEC group as the index $\left[\Lambda: \Gamma_{\sigma}\right]<+\infty$. In addition it is a surface group, so its signature has the form

$$
\left(h^{\prime} ; \pm ;[-] ;\left\{(-)^{k}\right\}\right. \text {, }
$$

which also determines the topological type of the symmetry $\sigma$, i.e. the sign decides about the separability, as it decides about the orientability of the orbit space $\mathcal{H} / \Gamma_{\sigma}$. Moreover, the number of period cycles is equal to the number of connected components 
of the boundary of this space and hence it is equal to the number of ovals of the symmetry $\sigma$. We shall use the theorem above taking $\tilde{\Lambda}=\Gamma_{\sigma}$, which will allow us to determine the separability character of the symmetry $\sigma$.

\subsection{Fixed points and the degree of hyperellipticity}

Let us recall that a Riemann surface $X$ is called $p$-hyperelliptic if it admits a conformal involution $\rho$ such that $X / \rho$ has topological genus $p$. This integer $\rho$ is called the hyperellipticity degree of $X$ with respect to $\rho$. Now, to retrieve the hyperellipticity degree of a surface it is convenient to use the famous Macbeath theorem from [14], which allows us to compute the number of points fixed by any conformal automorphism of $X$. The number of fixed points $m_{\rho}$ and the hyperellipticity degree $p_{\rho}$ of an involution $\rho$ of $X$ are connected by the formula $m_{\rho}=2 g+2-4 p_{\rho}$, so the values of $m_{\rho}$ and $p_{\rho}$ mutually determine each other. It is worthwhile mentioning that a similar result concerning automorphisms of Klein surfaces with fixed points was obtained in [3].

By $N_{G}(\langle g\rangle)$ we mean the normalizer in $G$ of the subgroup generated by $g$.

Theorem 2.4 (Macbeath [14]) Let $G=\Delta / \Gamma$ be the group of orientation preserving automorphisms of a Riemann surface $X=\mathcal{H} / \Gamma$ and let $x_{1}, x_{2}, \ldots, x_{r}$ be the set of canonical elliptic generators of $\Delta$ with proper periods $m_{1}, \ldots, m_{r}$ respectively. Let $\theta: \Lambda \rightarrow G$ be the canonical epimorphism. Then the number $m$ of points of $X$ fixed by $g \in G$ is given by the formula

$$
m=\left|N_{G}(\langle g\rangle)\right| \sum 1 / m_{i},
$$

where the sum is taken over those i for which $g$ is conjugate to a power of $\theta\left(x_{i}\right)$.

Note, that in the case of $s$-extremal Riemann surfaces we have 3 orientation preserving involutions in the automorphism group. Namely, if $G=\mathcal{D}_{n} \times \mathbb{Z}_{2}=\left\langle\sigma_{1}, \sigma_{2}\right.$ : $\left.\sigma_{1}^{2}=\sigma_{2}^{2}=\left(\sigma_{1} \sigma_{2}\right)^{n}\right\rangle \times\left\langle\tau: \tau^{2}\right\rangle$, then the orientation preserving involutions are $\sigma_{1} \tau, \sigma_{2} \tau$ and $\left(\sigma_{1} \sigma_{2}\right)^{n / 2}$.

\section{The button-like Riemann surfaces as the only extremal Riemann surfaces of even genera with one fixed point free symmetry}

We start with a simple example of a surface of genus 4 whose automorphism group is $G=\mathcal{D}_{4} \times \mathbb{Z}_{2}=\left\langle\sigma_{1}, \sigma_{2}\right\rangle \times\langle\tau\rangle$, admitting four non-conjugate symmetries $\sigma_{1}, \sigma_{2}, \tau, \tau\left(\sigma_{1} \sigma_{2}\right)^{2}$ of topological types $+1,+3,+5,0$ respectively.

Let $\Lambda$ be an NEC group with signature

$$
(0 ;+;[-] ;\{(2, . . ., 2,4)\})
$$

and we define an epimorphism $\theta: \Lambda \rightarrow G$ to map the consecutive canonical reflections of the only period cycle to

$$
\sigma_{1}, \tau, \sigma_{2}, \tau, \sigma_{2}, \sigma_{1}
$$




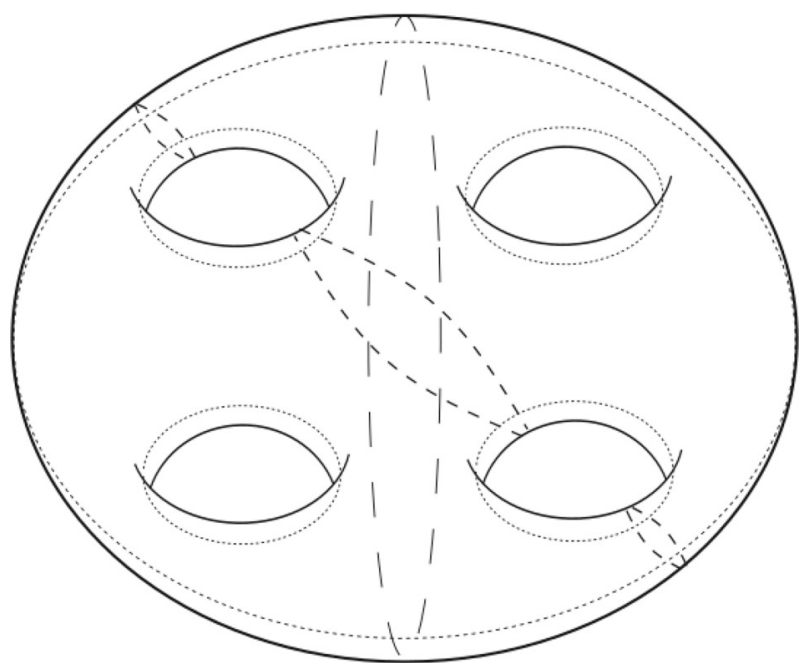

Fig. 1 The button-like Riemann surface of genus 4

Here, by the Hurwitz-Riemann formula we have

$$
\frac{g-1}{8}=-1+\frac{1}{2} \cdot\left(4 \cdot\left(1-\frac{1}{2}\right)+\left(1-\frac{1}{4}\right)\right)=\frac{1}{2}-\frac{1}{8}=\frac{3}{8}
$$

so indeed we obtain a Riemann surface $X=\mathcal{H} / \operatorname{ker} \theta$ of genus 4 . Now let us look closer at the topological types of symmetries $\sigma_{1}, \sigma_{2}, \tau$. We will find the number of ovals and separability character of the symmetry $\sigma_{2}$, as for the other symmetries we have the same argument.

Observe first, that the centralizer of $\sigma_{2}$ in $G$ has order 8. In addition, we have two reflections, $c_{2}$ and $c_{4}$, which are mapped to $\sigma_{2}$. By Theorem 2.2, the centralizer of $c_{2}$ in $\Lambda$ is generated by $c_{2}, c_{1} c_{2}$ and $c_{2} c_{3}$ and its image in $G$ is generated by $\sigma_{2}, \tau \sigma_{2}$, which is a subgroup of order 4 in $G$. Hence by Theorem 2.1, reflection $c_{2}$ contributes with $\frac{8}{4}=2$ ovals to $\left\|\sigma_{2}\right\|$. Now, the centralizer of reflection $c_{4}$ in $\Lambda$ is generated by $c_{4}, c_{3} c_{4},\left(c_{4} c_{0}\right)^{2}$ and so its image in $G$ is generated by $\sigma_{2}, \tau,\left(\sigma_{1} \sigma_{2}\right)^{2}$ and has order 8 . Therefore the contribution of $c_{4}$ to $\left\|\sigma_{2}\right\|$ is equal to $\frac{8}{8}=1$. This means that $\sigma_{2}$ has 3 ovals in total.

Now we shall check the separability character of $\sigma_{2}$ using Theorem 2.3. Let $\Gamma_{\sigma_{2}}=$ $\theta^{-1}\left(\left\langle\sigma_{2}\right\rangle\right)$. Of course we have $c_{2}, c_{4} \in \Gamma_{\sigma_{2}}$. Observe now that we have eight cosets

$$
\Gamma_{\sigma_{2}}, \Gamma_{\sigma_{2}} c_{0}, \Gamma_{\sigma_{2}} c_{1}, \Gamma_{\sigma_{2}} c_{0} c_{1}, \Gamma_{\sigma_{2}} c_{0} c_{2}, \Gamma_{\sigma_{2}} c_{0} c_{1} c_{2}, \Gamma_{\sigma_{2}} c_{0} c_{2} c_{0}, \Gamma_{\sigma_{2}}\left(c_{0} c_{2}\right)^{2} c_{1}
$$

These are the vertices of our Schreier coset graph. The edges are labelled with $c_{0}, c_{1}, c_{2}, c_{3}, c_{4}$, but it is easy to see that actually edges labelled by $c_{2}$ or $c_{4}$ are exactly the same and the same thing we have for edges $c_{1}, c_{3}$. The Schreier graph is presented in Fig. 2. 


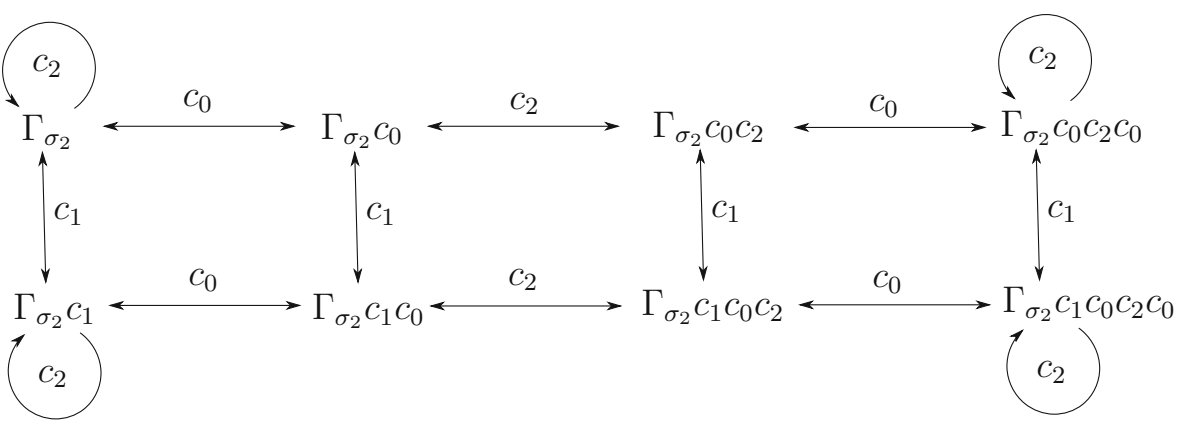

Fig. 2 Schreier coset graph

It is clear that the graph is bipartite and so all the circuits in our graph are positive and $\sigma_{2}$ is separating. As a matter of fact, the signature of $\Gamma_{\sigma_{2}}$ is

$$
(1 ;+;[-] ;\{(-),(-),(-)\})
$$

and the corresponding orbit space has topological genus 1 and its border consists of 3 connected components (ovals). In an analogous way we can show that $\sigma_{1}$ and $\tau$ are separating.

This example illustrates the simplest case of what we shall call a button-like surface. We later generalize it for any even integers $g, n$ such that $n$ divides $g$, where $g$ denotes the genus of the surface and $2 n$ is the order of the dihedral group generated by two non-central symmetries. Observe that, by Theorem 2.4 , the number of points fixed by the orientation preserving involutions are respectively $m_{\sigma_{1} \tau}=2, m_{\sigma_{2} \tau}=6$ and $m_{\left(\sigma_{1} \sigma_{2}\right)^{2}}=2$. Therefore, the corresponding hyperellipticity degrees are respectively $2,1,2$, which is concordant with our intuition while looking at how the respective involutions act on the surface (see Fig. 1).

Observe that the above surface bears many interesting properties, which justify our interest. First of all, we shall prove that this type of surface is an extremal Riemann surface, meaning that it admits the maximal possible number of non-conjugate symmetries together with the maximal number of ovals for 3 symmetries in the automorphism group in question. The third interesting property is that a Riemann surface of even genus can have at most three separating symmetries. We shall prove this result in the latter part of the paper, and the button-like surface is an interesting example of such a configuration, as it also involves a symmetry which attains the maximum number $g+1$ of ovals given by Harnack's theorem, [10].

In [6] Gromadzki and Izquierdo showed that a Riemann surface of even genus $g$ can admit at most 4 non-conjugate symmetries and the group of automorphisms is isomorphic to $G=\mathcal{D}_{n} \times \mathbb{Z}_{2}$ in such a case. In addition, they remarked in [7], that the total number of ovals of three non-conjugate symmetries generating $G$ is bounded from above by $\frac{2 g}{n}+g+2+\frac{n}{2}$ if $\frac{2 g}{n}$ is even and by $\frac{2 g}{n}+g+3$ if $\frac{2 g}{n}$ is odd. We shall show that in fact, if an extremal Riemann surface of even genus has one fixed point free symmetry, then $n$ divides $g$ and we propose a sharp bound in that case. Also, following the ideas of [7], as we look for the numbers of ovals of our symmetries and 
conjugate ones have the same numbers of ovals, we can actually assume, by Sylow Theorem, that our symmetries in fact generate a 2-group and so $n$ is a power of 2 .

Theorem 3.1 Let $g, n>2$ be even integers. Then, with the assumption that one of the symmetries is fixed point free, the 3 symmetries generating the automorphism group $\mathcal{D}_{n} \times \mathbb{Z}_{2}$ acting on a Riemann surface $X$ of genus $g$ can have at most $\frac{2 g}{n}+g+3$ ovals in total and the bound is attained if and only if $X$ is a button-like surface.

Proof Let $g, n>2$ be integers as in the theorem and let $G=\mathcal{D}_{n} \times \mathbb{Z}_{2}=\left\langle\sigma_{1}, \sigma_{2}\right\rangle \times\langle\tau\rangle$ be the automorphism group of a Riemann surface $X=\mathcal{H} / \Gamma$, where $\Gamma$ is the kernel of a smooth epimorphism from an NEC group $\Lambda$ with general signature (1) to $G$. We shall look for the numbers of ovals of symmetries $\sigma_{1}, \sigma_{2}, \tau$. Observe that there is an odd number of link periods equal $n$ in the signature of $\Lambda$. Indeed, $g$ is an even integer, and so we have that $\frac{g-1}{2 n}$ is a fraction with odd numerator and denominator equal to $2 n$, as it cannot be reduced. Now by the Hurwitz-Riemann formula we have

$$
\frac{g-1}{2 n}=\frac{\mu(\Lambda)}{2 \pi}
$$

and both sides can be seen as fractions with denominator $2 n$ and so the right-hand side also has an odd numerator. But it basically means that the number of link periods equal to $n$ in the signature of $\Lambda$ is odd. Hence there is at least one non-empty period cycle in the signature of $\Lambda$.

First of all, we shall prove that there exists a canonical reflection $c$, with $\theta(c)=\tau$ such that the neighboring reflections of $c$ have images, without loss of generality, equal to $\sigma_{1}$ and $\sigma_{2}$. Here we treat reflections in the non-empty period cycle as situated on a circle. Observe, that there is a non-empty period cycle which involves, without loss of generality, $\sigma_{1}, \sigma_{2}$ and $\tau$. Indeed, if the period cycle involves only symmetries $\sigma_{1}, \sigma_{2}$ and their conjugates, then the number of link periods equal $n$ in such a cycle must be even, as the first and the last reflection in the cycle are conjugate and a link period equal $n$, being a power of 2 , only arises as a product of a symmetry conjugate to $\sigma_{1}$ and a symmetry conjugate to $\sigma_{2}$. So, there exists a non-empty period cycle involving all three symmetries. We may assume that the cycle starts with the symmetry $\sigma_{1}$ and the first link period is $n$. Observe that we can only skip from a conjugate of $\sigma_{1}$ to a conjugate of $\sigma_{2}$, without using $\tau$, by using a link period $n$. Therefore in the beginning we have some sequence of link periods $n$, we may assume that their number is odd, so the sequence starts with $\sigma_{1}$ and finishes with $\sigma_{2}$. Then, after perhaps some sequence of symmetries conjugate to $\sigma_{2}$, a symmetry $\tau$ must appear. If its right-hand side neighbor is a symmetry conjugate to $\sigma_{1}$, we finish the procedure. If not, then we have to continue until a symmetry conjugate to $\sigma_{1}$ appears. If it appears right after $\tau$, then we finish the procedure. If it appears after a symmetry conjugate to $\sigma_{2}$, then again we start a sequence of link periods equal $n$ and we repeat the previous steps. If we reach a symmetry $\tau$ with neighbors conjugate to $\sigma_{1}$ and $\sigma_{2}$, we finish the procedure. So let us assume that we reach the last link period $n$ and such a situation did not happen. As there is an odd number of link periods equal $n$, and we started with $\sigma_{1}$, the righthand side neighbor of the last link period $n$ must be conjugate to $\sigma_{2}$. Now there are no more link periods equal $n$ which we may use, so we proceed with a sequence of 
symmetries conjugate to $\sigma_{2}$ and a symmetry $\tau$. As the first and last reflection of the cycle are conjugate and the image of the first reflection was $\sigma_{1}$, a symmetry conjugate to $\sigma_{1}$ must appear. As it cannot appear after a symmetry conjugate to $\sigma_{2}$ - we have no more link periods equal $n$-it must appear after a symmetry $\tau$ and again we finish the procedure. Summing up, we have proved that there is at least one appearance of a symmetry $\tau$ with neighbors conjugate to $\sigma_{1}, \sigma_{2}$. Now by Theorems 2.1 and 2.2 observe, that this particular reflection with image $\tau$ contributes with only 1 oval. We shall use this fact in the latter part of the proof.

Now we shall prove the bound on the total number of ovals of the symmetries $\sigma_{1}, \sigma_{2}$ and $\tau$, proposed in the statement. By the Hurwitz-Riemann formula there exists a nonnegative integer $s$ such that

$$
\frac{g-1}{2 n}=\frac{\mu(\Lambda)}{2 \pi} \geq-1+l+\frac{s}{4}+\frac{1}{2}-\frac{1}{2 n}
$$

and in turn

$$
s \leq \frac{2 g}{n}-4 l+2 .
$$

Suppose first that $l>0$. By Theorems 2.1 and 2.2, a central symmetry of the nonempty period cycle contributes at most $n$ ovals if its neighbors coincide and at most $n / 2$ ovals if its neighbors are distinct. On the other hand, the number of ovals of a central symmetry connected to an empty period cycle is at most $2 n$, whereas the corresponding values for a non-central symmetry are, respectively, 2, 1 or 4 .Thus, the total number $\|X\|$ of ovals of the surface $X$ satisfies the inequality

$$
\|X\| \leq(s / 2-1) \cdot n+1+(s / 2-1) \cdot 2+2+2 n l,
$$

as there is an appearance of $\tau$ which contributes only one oval and there are at least two appearances of $\sigma_{1}$ and $\sigma_{2}$, connected with link periods $n$, which contribute one oval to both of the symmetries. Now, using the inequality (5) on $s$, we obtain $\|X\| \leq$ $(s / 2-1)(n+2)+3+2 n l \leq g+\frac{2 g}{n}+3-4 l<g+\frac{2 g}{n}+3$ and the proof in this case is finished.

Now let us assume that $l=0$ in the signature of $\Lambda$. Then inequality (5) says

$$
s \leq \frac{2 g}{n}+2, \text { that is, } \frac{s}{2}-1 \leq \frac{g}{n},
$$

and again

$$
\|X\| \leq(s / 2-1) \cdot n+1+(s / 2-1) \cdot 2+2 \leq g+1+\frac{2 g}{n}+2=g+\frac{2 g}{n}+3 .
$$

Finally, assume that $l=0, k=1$ in the signature of $\Lambda$. In such a case

$$
s \leq \frac{2 g}{n}+2
$$


and the equality holds if and only if $h=r=0$ in the signature of $\Lambda$ and all but one link periods are equal 2 . Then

$$
\|X\| \leq(s / 2-1) n+1+(s / 2-1) 2+2 \leq g+\frac{2 g}{n}+3 .
$$

Now observe that if the bound is attained, then the signature of $\Lambda$ is exactly

$$
(0 ;+;[-] ;\{(2, . s ., 2, n)\}),
$$

where $s=\frac{2 g}{n}+2$. In addition, to have equality in (6), we have to take care that the epimorphism $\theta: \Lambda \rightarrow G=\mathcal{D}_{n} \times \mathbb{Z}_{2}=\left\langle\sigma_{1}, \sigma_{2}\right\rangle \times\langle\tau\rangle$ is defined so that exactly one appearances of symmetries $\sigma_{1}, \sigma_{2}, \tau$ contribute with 1 oval and the other with 2 or $n$ ovals respectively, which basically means, that except of these three special appearances, all the symmetries have neighbors with the same images under $\theta$. Hence, without loss of generality, the epimorphism $\theta: \Lambda \rightarrow G$ has to map the consecutive canonical reflections of the only period cycle to

$$
\underbrace{\sigma_{1}, \tau, \ldots, \sigma_{1}, \tau}_{2 \alpha}, \underbrace{\sigma_{2}, \tau, \ldots, \sigma_{2}, \tau}_{2 \beta}, \sigma_{2}, \sigma_{1}
$$

for some nonnegative integers $\alpha$ and $\beta$. Here clearly $2 \alpha+2 \beta=\frac{2 g}{n}+2$, hence $\frac{2 g}{n}$ must be even. By the usual methods of computing centralizers in $G$ and in $\Lambda$ given in Theorems 2.1 and 2.2, we can easily calculate that:

$$
\begin{aligned}
& \|\tau\|=(\alpha-1) \cdot n+1+\beta n=g+1,\left\|\sigma_{1}\right\|=(\alpha-1) \cdot 2+1=2 \alpha-1, \\
& \left\|\sigma_{2}\right\|=\beta \cdot 2+1=2 \beta+1
\end{aligned}
$$

It is important for us that

$$
\left\|\sigma_{1}\right\|+\left\|\sigma_{2}\right\|=2(\alpha+\beta)=\frac{2 g}{n}+2
$$

One can also easily check, by the results concerning Schreier coset graph method, that $\sigma_{1}, \sigma_{2}, \tau$ are all separating —in fact $\tau$ must be separating as it has $g+1$ ovals. For $\sigma_{1}$ and $\sigma_{2}$ we can draw a bipartite Schreier coset graph similar to the one in Fig. 2, but with $2 n$ cosets. Obviously the surface described above holds the conditions announced in the theorem and it is unique in a sense of uniqueness of the class of epimorphisms. This means that there is exactly one connected family of extremal surfaces in the conditions of Theorem 3.1. The real dimension of the family is $s-2 \geq 1$. From now on such a surface shall be called a button-like Riemann surface. On Fig. 3 we see a button-like Riemann surface with automorphism group $\mathcal{D}_{8} \times \mathbb{Z}_{2}$ with $\alpha=\beta=2$.

Corollary 3.2 The hyperellipticity degrees of the involutions $\sigma_{1} \tau, \sigma_{2} \tau,\left(\sigma_{1} \sigma_{2}\right)^{n / 2}$ for the button-like surface in the above theorem are respectively $\frac{g}{2}+1-\alpha, \frac{g}{2}-\beta$ and $\frac{g}{2}$. 


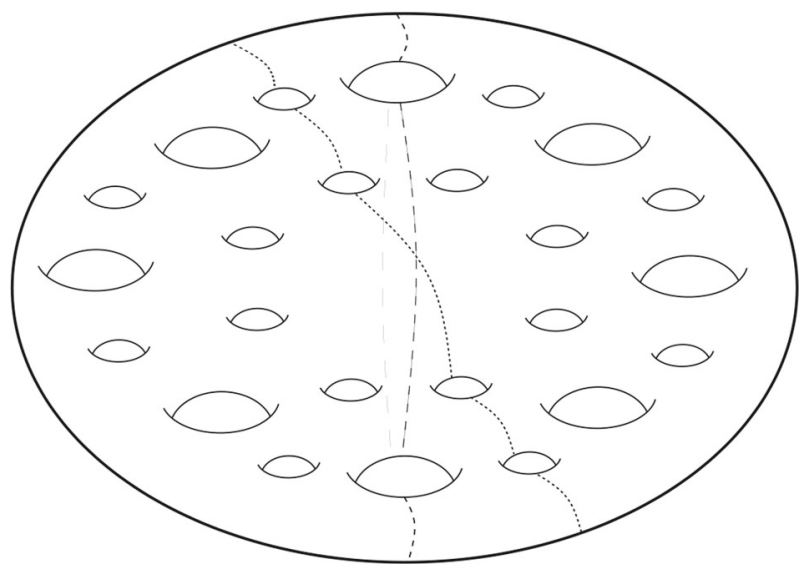

Fig. 3 A button-like Riemann surface of genus 24

Proof We should use the unique epimorphism (8) and Theorem 2.4. By Theorem 2.4 we can easily see that the number of points fixed by $\sigma_{1} \tau, \sigma_{2} \tau,\left(\sigma_{1} \sigma_{2}\right)^{n / 2}$ is respectively equal to $4 \alpha-2,4 \beta+2$ and 2 , as every appearance of a product $\sigma_{1} \tau$ or $\sigma_{2} \tau$ contributes with 2 fixed points and the appearance of $\left(\sigma_{1} \sigma_{2}\right)^{n / 2}$ contributes with 2 fixed points as well. Therefore, by using the formula $m=2 g+2-4 p$ we see that $p_{\sigma_{1} \tau}=\frac{g}{2}+1-\alpha$, $p_{\sigma_{2} \tau}=\frac{g}{2}-\beta$ and $p_{\left(\sigma_{1} \sigma_{2}\right)^{n / 2}}=\frac{g}{2}$, where as before $\alpha+\beta=\frac{g}{n}+1$.

Remark 3.3 One might ask what happens if we assume all the symmetries to have fixed points. By [7] it is known, that the bound on the total number of ovals is $2 g+2$. This situation is, however, less interesting, as it was proven in [12] that the only admissible symmetry type for such an extremal Riemann surface is $\{+1,+1,-g,-g\}$. It is not difficult to obtain the degree of hyperellipticity of the involutions in the automorphism group. As before, the respective numbers of fixed points $m_{\rho}$ are 2, 2, $2 g+2,2 g+2$ and so the hyperellipticity degrees can be easily calculated and are equal to $\frac{g}{2}, \frac{g}{2}, 0,0$.

\section{Some properties of s-extremal Riemann surfaces of even genus}

In this part of the paper we show some results concerning the possible configurations of topological types of symmetries on an $s$-extremal Riemann surface.

Theorem 4.1 A Riemann surface of even genus $g$ has at most 3 separating symmetries.

Proof It is enough to show that if a Riemann surface of even genus $g$ is $s$-extremal, that is, it admits 4 non-conjugate symmetries, then at least 1 of them must be nonseparating. So let us assume that $X=\mathcal{H} / \Gamma$ is a Riemann surface of even genus $g$, admitting 4 non-conjugate symmetries. In such a case, by the results of [6] we know that its automorphism group is $G=\mathcal{D}_{n} \times \mathbb{Z}_{2}=\left\langle\sigma_{1}, \sigma_{2}\right\rangle \times\langle\tau\rangle$. Let $\Lambda$ be an NEC group with general signature (1) such that $G=\Lambda / \Gamma$ and let $\theta: \Lambda \rightarrow G$ be the canonical epimorphism. We have 4 non-conjugate symmetries $\sigma_{1}, \sigma_{2}, \tau, \tau\left(\sigma_{1} \sigma_{2}\right)^{n / 2}$ in $G$. If all of these have fixed points, then there exist canonical reflections $c_{\sigma_{1}}, c_{\sigma_{2}}, c_{\tau}, c_{\tau\left(\sigma_{1} \sigma_{2}\right)^{n / 2}}$ 
mapped respectively to the symmetries above. We also have the corresponding NEC subgroups $\Gamma_{\sigma_{1}}, \Gamma_{\sigma_{2}}, \Gamma_{\tau}, \Gamma_{\tau\left(\sigma_{1} \sigma_{2}\right)^{n / 2}}$ of $\Lambda$, defined as described in Preliminaries. Now observe that for each of the symmetries, the corresponding Schreier coset graph has negative circuits. For example, let us consider the symmetry $\tau\left(\sigma_{1} \sigma_{2}\right)^{n / 2}$. The negative circuit is produced by following the path labelled by a sequence of reflections

$$
c_{\tau} \underbrace{c_{\sigma_{1}} c_{\sigma_{2}}}_{n / 2} .
$$

Hence clearly $\tau\left(\sigma_{1} \sigma_{2}\right)^{n / 2}$ is non-separating. Similarly one can built a negative circuit in a Schreier coset graph corresponding to $\tau$.

The previous result leads to the following interesting corollaries.

Corollary 4.2 If X is a Riemann surface of even genus $g$ admitting four non-conjugate symmetries with fixed points, then at least two of them are central and non-separating.

Proof The first part follows easily by the results of [6], as we know that if a Riemann surface $X$ of even genus admits 4 non-conjugate symmetries, then $\operatorname{Aut}(X)=\mathcal{D}_{n} \times \mathbb{Z}_{2}$. Therefore at least two of the symmetries in question are central. Now, as all the symmetries have fixed points, then by the proof of the previous theorem both of these central symmetries must be non-separating.

Corollary 4.3 If $X$ is a Riemann surface of even genus $g$ admitting four non-conjugate symmetries, and one of them has $g+1$ ovals, then one of the remaining symmetries is fixed-point free.

Proof If $X$ admits a symmetry with the maximal number of $g+1$ ovals, then the symmetry must be central and separating. The second statement is obvious. As for the first one, observe that a non-central symmetry can have at most $g / 2+1$ ovals. Indeed, by the results of [2], the bound for the total number of ovals of two symmetries is $4 g / n+2$, where $n$ denotes the order of their product. Hence, if we assume, by way of contradiction, that a non-central symmetry has $g+1$ ovals here, then $n=4$ and the other non-central symmetry has only 1 oval. Nevertheless, using the notations from the proof of Theorem 3.1, we may show in an analogous way, that the number of ovals of a non-central symmetry is bounded from above by

$$
(s / 2-1) 2+1+4 l \leq g / 2+1
$$

as $s \leq g / 2+2$. Therefore the symmetry with $g+1$ ovals must be central in our case. Now if the other central symmetry would have fixed points, then by the previous corollary, both of the central symmetries would be non-separating, a contradiction.

Observe that, as a byproduct of the previous proof, we have showed

Corollary 4.4 A non-central symmetry on a Riemann surface of even genus g, admitting four non-conjugate symmetries, has at most $g / 2+1$ ovals. 
Next we prove some results concerning surfaces admitting a symmetry with $g+1$ ovals. Observe, that by Corollary 4.3 , such a symmetry is unique in the automorphism group if there are non-central symmetries in the group, which is our case here.

Proposition 1 A Riemann surface of even genus $g$ whose automorphism group is $G=$ $\mathcal{D}_{n} \times \mathbb{Z}_{2}$ for some even integer $n>2$ admits a symmetry with $g+1$ ovals if and only if $g$ is divisible by $n$.

Proof For the sufficient condition let $b=\frac{g}{n}+1$, that is a positive integer since $g$ is divisible by $n$, and let $\Lambda$ be an NEC group with signature

$$
(0 ;+;[-] ;\{(2, .2 b, 2, n)\}) .
$$

Consider an epimorphism $\theta: \Lambda \rightarrow G=\left\langle\sigma_{1}, \sigma_{2}\right\rangle \times\langle\tau\rangle$ which maps the consecutive canonical reflections $c_{0}, \ldots, c_{2 b+1}$ corresponding to the unique period cycle in the signature of $\Lambda$ to

$$
\sigma_{1}, \tau, \ldots, \sigma_{1}, \tau, \sigma_{2}, \sigma_{1}
$$

The Riemann surface $X=\mathcal{H} / \operatorname{ker} \theta$ has genus $n(b-1)=g$ and, by Theorems 2.1 and 2.2, $\tau$ is a symmetry of $X$ with $\|\tau\|=g+1$ ovals, as announced in the statement. Observe that in fact this is a button-like surface. However, these are not the only surfaces that admit a symmetry whose number of ovals is maximum.

Conversely, let $X$ be a Riemann surface of even genus $g$ whose automorphism group is the group $G$ in the statement, admitting a symmetry $\tau$ with $g+1$ ovals. Let also $\Lambda$ be an NEC group with general signature (1). By the Hurwitz-Riemann formula there exists a non-empty period cycle in the signature of $\Lambda$ with an odd number of link periods equal to $n$. By the proof of Theorem 3.1 the symmetry $\tau$ appears at least once as the image of a canonical reflection $c_{i} \in \Lambda$. We may assume, without loss of generality, that $\sigma_{1}$ and $\sigma_{2}$ are the images of the neighbors $c_{i-1}$ and $c_{i+1}$ of $c_{i}$. Moreover, by the proof of Theorem 3.1 the symmetry $\tau$ appears at least once as an image of the canonical reflection with neighbors, without loss of generality, $\sigma_{1}, \sigma_{2}$. Observe that by Theorems 2.1 and 2.2, an empty period cycle contributes at most $2 n$ ovals to the central symmetry with $g+1$ ovals. Let $s$ denote the number of link periods equal 2 - these are the only ones interesting for us, as they can involve a central symmetry. Hence again

$$
\|\tau\| \leq\left(\frac{s}{2}-1\right) n+1+2 n l
$$

and by the Hurwitz-Riemann formula $l+\frac{s}{4} \leq \frac{g}{2 n}+\frac{1}{2}$ and the equality holds if and only if $h=r=0$ in the signature of $\Lambda, k=1$ and all the link periods except one are equal 2 . Observe that the equality $\|\tau\|=g+1$ implies that the only non-empty period cycle is of the form $(2, \ldots, 2, n)$ where the consecutive canonical reflections are mapped as in the case of the button-like surface, that is (8). In addition, all the connecting generators are mapped to 1 and the reflections of the empty period cycles 
to $\tau$. It also follows that $s=2 b$ is even and so $g=n(b+l-1)$. Observe that in the special case $l=0$ we obtain exactly the button like-surface.

\section{The surface of minimal genus}

Along this section we fix an even integer $n>2$ and denote $\Sigma_{n}$ the set of all (compact) Riemann surfaces whose automorphism group is $\mathcal{D}_{n} \times \mathbb{Z}_{2}$. Let $g_{n}$ be the minimum of the genera of those $X \in \Sigma_{n}$ whose genus is even.

Theorem 5.1 We have:

(1) $g_{n}=n / 2$.

(2) If $X \in \Sigma_{n}$ has genus $g_{n}$, then $X$ is hyperelliptic. In addition, $X$ admits two symmetries with 1 oval and a symmetry with 3 ovals.

(3) Let $g$ be a positive even integer divisible by $n / 2$. Then there exists $Y \in \Sigma_{n}$ whose genus equals $g$.

Proof Along the proof we fix two elements $\sigma_{1}, \sigma_{2}$ of order 2 in the dihedral group $\mathcal{D}_{n}$ whose product has order $n$, and a generator $\tau$ of the cyclic group $\mathbb{Z}_{2}$.

(1) Let $\Lambda_{0}$ be an NEC group whose signature is $(0 ;+;[-] ;\{(2,2,2, n)\})$ and denote $c_{0}, c_{1}, c_{2}, c_{3}, c_{4}$ the consecutive canonical reflections of $\Lambda_{0}$ corresponding to the unique period cycle in the signature of $\Lambda_{0}$. Then, the assignment

$$
c_{0} \rightarrow \sigma_{1}, c_{1} \rightarrow \tau, c_{2} \rightarrow \sigma_{2}\left(\sigma_{1} \sigma_{2}\right)^{n / 2}, c_{3} \rightarrow \sigma_{2} \text { and } c_{4} \rightarrow \sigma_{1}
$$

induces an epimorphism $\theta_{0}: \Lambda_{0} \rightarrow \mathcal{D}_{n} \times \mathbb{Z}_{2}$ whose kernel $\Gamma_{0}$ is a surface NEC group. Thus, $X=\mathcal{H} / \Gamma_{0} \in \Sigma_{n}$. Let us compute the genus $g$ of $X$. By the Hurwitz-Riemann formula,

$$
\mu\left(\Lambda_{0}\right)=2 \pi \cdot\left(\frac{g-1}{2 n}\right)
$$

On the other hand,

$$
\mu\left(\Lambda_{0}\right)=2 \pi\left(-1+\frac{1}{2}\left(3 \cdot\left(1-\frac{1}{2}\right)+1-\frac{1}{n}\right)\right)=2 \pi\left(\frac{1}{4}-\frac{1}{2 n}\right)
$$

Consequently,

$$
\frac{g-1}{2 n}=\frac{1}{4}-\frac{1}{2 n}
$$

so $g=\frac{n}{2}$. This proves that $g_{n} \leq \frac{n}{2}$. To prove the converse inequality, let $Y \in \Sigma_{n}$. Then there exist an NEC group $\Lambda$ and an epimorphism $\theta: \Lambda \rightarrow \mathcal{D}_{n} \times \mathbb{Z}_{2}$ whose kernel $\Gamma$ is a surface NEC group and $Y=\mathcal{H} / \Gamma$. Let $g$ be the genus of $Y$. By the 
Hurwitz-Riemann formula,

$$
\mu(\Lambda)=2 \pi \cdot\left(\frac{g-1}{2 n}\right)
$$

and, as $g-1$ is odd, there must be an odd number of link periods equal $n$ in the signature of $\Lambda$. As the surface $Y$ has three nonconjugate symmetries with ovals, the signature of $\Lambda$ has, at least, three link periods equal 2 . Therefore,

$$
2 \pi \cdot\left(\frac{g-1}{2 n}\right) \geq 2 \pi\left(-1+\frac{1}{2}\left(3 \cdot\left(1-\frac{1}{2}\right)+1-\frac{1}{n}\right)\right)=2 \pi\left(\frac{1}{4}-\frac{1}{2 n}\right)
$$

and $g \geq n / 2=g_{n}$. This proves the first part in the statement.

(2) Let $X \in \Sigma_{n}$ with genus $g_{n}$. The number $m$ of points in $X$ fixed by the orientation preserving automorphism $\gamma=\left(\sigma_{1} \sigma_{2}\right)^{n / 2}$ is, by the well-known Macbeath theorem in [14], $m=n+2=2 g_{n}+2$. As the hyperellipticity degree $p$ of $X$ with respect to $\gamma$ satisfies $m=2 g_{n}+2-4 p$ it follows that $p=0$, that is, $X$ is hyperelliptic. Note in addition that, by Theorems 2.1 and 2.2, the symmetries $\sigma_{1}$ and $\tau$ of $X$ have 1 oval, whereas the symmetry $\sigma_{2}$ has 3 ovals.

(3) Let $a \geq 2$ be an integer such that $g=a n / 2$ and let $\Lambda$ be an NEC group with signature

$$
(0 ;+;[-] ;\{(2, \stackrel{a+2}{\cdot}, 2, n)\})
$$

and let $\theta: \Lambda \rightarrow \mathcal{D}_{n} \times \mathbb{Z}_{2}$ be the epimorphism which maps, respectively, the consecutive canonical reflections corresponding to the unique period cycle of the signature of $\Lambda$ to

$$
\underbrace{\sigma_{1}, \tau, \ldots, \sigma_{1}, \tau}_{a+1}, \sigma_{2}\left(\sigma_{1} \sigma_{2}\right)^{n / 2}, \sigma_{2}, \sigma_{1}
$$

if $a$ is odd and

$$
\underbrace{\sigma_{1}, \tau, \ldots, \sigma_{1}, \tau,}_{a+2} \sigma_{2}, \sigma_{1}
$$

if $a$ is even. Then $Y=\mathcal{H} / \operatorname{ker} \theta \in \Sigma_{n}$ has genus $g$ and we are done.

Remark 5.2 Defining equations of the hyperelliptic surface $X \in \Sigma_{n}$ of genus $n / 2$ constructed in the theorem above can be found in [1], as it is a hyperelliptic surface. In addition, a different approach was presented also in [16], where the case $n=4$ is treated in detail. 


\section{Surface with the minimal number of ovals}

As in the precedent section we denote $\Sigma_{n}$, for every even integer $n>2$, the set of all Riemann surfaces whose automorphism group is $\mathcal{D}_{n} \times \mathbb{Z}_{2}$.

In [9] we have considered the minimal total number of ovals that a maximal set of symmetries can admit. In case of even $g$ the maximal number of symmetries is 4 and we proved that in fact 4 symmetries, with fixed points must have at least 5 ovals and the bound is sharp. However, one can consider the minimal number of ovals for the symmetries in the automorphism group $\mathcal{D}_{n} \times \mathbb{Z}_{2}$, and let some of them to be fixed point free.

Proposition 2 Let $n$ be an even integer, let $\sigma_{1}$ and $\sigma_{2}$ be generators of order 2 of the dihedral group $\mathcal{D}_{n}$ whose product has order $n$ and let $\tau$ be a generator of the cyclic group $\mathbb{Z}_{2}$ of order 2 .

(1) Let $X \in \Sigma_{n}$ with even genus. Then $\left\|\sigma_{1}\right\|+\left\|\sigma_{2}\right\|+\|\tau\| \geq 3$.

(2) For every integer $g \in n \mathbb{Z}$ with $g \geq 2$ there exists a surface $X \in \Sigma_{n}$ of genus $g$ such that $\left\|\sigma_{1}\right\|+\left\|\sigma_{2}\right\|+\|\tau\|=3$.

Proof (1) As $X \in \Sigma_{n}$ there exist an NEC group $\Lambda$ and an epimorphism $\theta: \Lambda \rightarrow$ $\mathcal{D}_{n} \times \mathbb{Z}_{2}$ whose kernel is a surface NEC subgroup $\Gamma$ such that $X=\mathcal{H} / \Gamma$. Let $g$ be the genus of $X$. By the Hurwitz-Riemann formula,

$$
\mu(\Lambda)=2 \pi\left(\frac{g-1}{2 n}\right)
$$

and, since $g$ is even, the number of link periods in the signature of $\Lambda$ equal to $n$ is odd. As $\sigma_{1}$ and $\sigma_{2}$ are not conjugate, $n \geq 4$. In particular the only non-empty period cycle in the signature of $\Lambda$ is not $(n, n)$. Thus, it does not happen that $\tau\left(\sigma_{1} \sigma_{2}\right)^{n / 2}$ and $\tau$ are fixed-point free and $\left\|\sigma_{1}\right\|=\left\|\sigma_{2}\right\|=1$, which is the unique situation with $\left\|\sigma_{1}\right\|+\left\|\sigma_{2}\right\|+\|\tau\|<3$.

(2) Let $a=g / n$ and choose an NEC group $\Lambda$ whose signature is

$$
(0 ;+;[2, . a, 2] ;\{(2,2, n)\}) .
$$

Let $x_{1}, \ldots, x_{a}$ be the canonical elliptic generators of $\Lambda, e_{1}$ the connecting generator and $c_{0}, c_{1}, c_{2}, c_{3}$ its canonical reflections. Let $\theta: \Lambda \rightarrow \mathcal{D}_{n} \times \mathbb{Z}_{2}$ be the epimorphism satisfying

$\theta\left(x_{i}\right)=\left(\sigma_{1} \sigma_{2}\right)^{n / 2}$ for $i=1, \ldots, a, \theta\left(c_{0}\right)=\sigma_{1}, \theta\left(c_{1}\right)=\tau, \theta\left(c_{2}\right)=\sigma_{2}, \theta\left(c_{3}\right)=\sigma_{1}$

and

$$
\theta\left(e_{1}\right)= \begin{cases}1 & \text { if } a \text { is even } \\ \left(\sigma_{1} \sigma_{2}\right)^{n / 2} & \text { if } a \text { is odd }\end{cases}
$$


Then $X \in \Sigma_{n}$ and if $g$ denotes the genus of $X$ we have, by the Hurwitz-Riemann formula,

$$
\frac{g-1}{2 n}=\frac{a}{2}-\frac{1}{2 n}
$$

that is, $g=a n$. Finally, $\left\|\sigma_{1}\right\|=\left\|\sigma_{2}\right\|=1$, so $\left\|\sigma_{1}\right\|+\left\|\sigma_{2}\right\|+\|\tau\|=3$.

\section{The case of 5 symmetries}

Although the paper focuses on Riemann surfaces of even genus, there is a common trait with the surfaces having 5 symmetries. Namely, the values 4 and 5 are the only ones that can be realized with a non-abelian automorphism group. Therefore here, as the last part of the paper, we find the possible topological types of the symmetries for an $o$-extremal Riemann surface admitting 5 non-conjugate symmeries, two of which are non-central.

Note that the sharp upper bound on the number of ovals of 5 non-conjugate symmetries of a Riemann surface was found in [8]. In the paper it was also stated (see Corollary 4.1), that the group generated by the 5 symmetries on a $o$-extremal Riemann surface is isomorphic to

$$
\mathcal{D}_{n} \times \mathbb{Z}_{2}^{3}=\left\langle\sigma_{1}, \sigma_{2}: \sigma_{1}^{2}=\sigma_{2}^{2}=\left(\sigma_{1} \sigma_{2}\right)^{n}\right\rangle \times\left\langle\tau_{1}: \tau_{1}^{2}\right\rangle \times\left\langle\tau_{2}: \tau_{2}^{2}\right\rangle \times\left\langle\tau_{3}: \tau_{3}^{2}\right\rangle .
$$

Of course if we take $n=2$ then we obtain a configuration of commuting symmetries and the possible topological types are already found in [13]. So let us assume that in fact $n \geq 4$. Let $X=\mathcal{H} / \Gamma$ be an $o$-extremal Riemann surface of genus $g$, having 5 symmetries generating the group $G=\mathcal{D}_{n} \times \mathbb{Z}_{2}^{3}$. We know that $G=\Lambda / \Gamma$ for some NEC group $\Lambda$ and a Fuchsian surface group $\Gamma$. We also have the canonical epimorphism $\theta: \Lambda \rightarrow G$. By the results of [8] we know that in fact the signature of $\Lambda$ is of the form

$$
(0 ;+;[-] ;\{(2, . . ., 2)\}),
$$

where $s=\frac{g-1}{2 n}+1$. In addition, for the symmetries to have a maximal total number of ovals, it must be that non-central symmetries $\sigma_{1}, \sigma_{2}$ (or their conjugates, to be precise), appear only once in the sequence of images of consecutive canonical reflections. Moreover, they have central neighbors and there is no central symmetry with two noncentral neighbors. In addition, the number of symmetries with distinct neighbors is exactly 4 . Therefore these 4 must be exactly neighbors of our non-central symmetries. Summing up, our epimorphism has the general form:

$$
\tau_{1} \sigma_{1} \underbrace{\tau_{1} \tau_{2} \tau_{1} \ldots \tau_{2}}_{2 \alpha} \tau_{1} \sigma_{2} \underbrace{\tau_{1} \tau_{3} \tau_{1} \ldots \tau_{3}}_{2 \beta} \tau_{1}
$$

as this is the only possible way we can satisfy the above conditions. It can be easily seen that all the central images of canonical reflections with the same neighbors contribute $\frac{16 n}{4}=4 n$ ovals each, the non-central entries contribute 8 ovals each and the central entries with distinct neighbors contribute $2 n$ ovals each. Hence in total we have 


$$
8 n \alpha+8 n \beta+16=2 g+14
$$

ovals, which is the maximum value we expected. Also, by the results of [11] we easily see that all the symmetries are separating. Therefore we can conclude with the following

Theorem 7.1 If a Riemann surface $X_{g}$ admits five non-conjugate symmetries generating a non-abelian group $\mathcal{D}_{n} \times \mathbb{Z}_{2}^{3}$ with a maximal total number of $2 g+14$ ovals, then their topological types are

$$
\{+8,+8,+4 n \alpha,+4 n \beta,+4 n(\alpha+\beta)\},
$$

where $4 n \alpha+4 n \beta+1=g$.

Open Access This article is licensed under a Creative Commons Attribution 4.0 International License, which permits use, sharing, adaptation, distribution and reproduction in any medium or format, as long as you give appropriate credit to the original author(s) and the source, provide a link to the Creative Commons licence, and indicate if changes were made. The images or other third party material in this article are included in the article's Creative Commons licence, unless indicated otherwise in a credit line to the material. If material is not included in the article's Creative Commons licence and your intended use is not permitted by statutory regulation or exceeds the permitted use, you will need to obtain permission directly from the copyright holder. To view a copy of this licence, visit http://creativecommons.org/licenses/by/4.0/.

\section{References}

1. Bujalance, E., Cirre, F.J., Gamboa, J.M., Gromadzki, G.: Symmetry types of hyperelliptic Riemann surfaces. Mém. Soc. Math. Fr. 86, vi+122 pp (2001)

2. Bujalance, E., Costa, A.F., Singerman, D.: Application of Hoare's theorem to symmetries of Riemann surfaces. Ann. Acad. Sci. Fenn. 18, 307-322 (1993)

3. Corrales, C., Gamboa, J.M., Gromadzki, G.: Automorphisms of Klein surfaces with fixed points. Glasg. Math. J. 41(2), 183-189 (1999)

4. Estévez, J.L., Izquierdo, M.: Non-normal pairs of non-Euclidean crystallographic groups. Bull. Lond. Math. Soc. 38, 113-123 (2006)

5. Gromadzki, G.: On a Harnack-Natanzon theorem for the family of real forms of Riemann surfaces. J. Pure Appl. Algebra 121, 253-269 (1997)

6. Gromadzki, G., Izquierdo, M.: Real forms of a Riemann surface of even genus. Proc. Am. Math. Soc. 126, 3475-3479 (1998)

7. Gromadzki, G., Izquierdo, M.: On ovals of Riemann surfaces of even genera. Geom. Dedicata 78, 81-88 (1999)

8. Gromadzki, G., Kozłowska-Walania, E.: On ovals of non-conjugate symmetries of Riemann surfaces. Int. J. Math. 20, 1-13 (2009)

9. Gromadzki, G., Kozłowska-Walania, E.: The groups generated by maximal sets of symmetries of Riemann surfaces and extremal quantities of their ovals. Mosc. Math. J. 18(3), 421-436 (2018)

10. Harnack, A.: Über die Vieltheiligkeit der ebenen algebraischen Curven. Math. Ann. 10, 189-199 (1876)

11. Hoare, A.H.M., Singerman, D.: The Orientability of Subgroups of Plane Groups. Groups St. Andrews 1981 (St. Andrews, 1981), London Mathematical Society Lecture Note series, vol. 71, pp. 221-227. Cambridge University Press, Cambridge (1982)

12. Kozłowska-Walania, E.: Extremal configurations of three or four symmetries on a Riemann surface. Bull. Kor. Math. Soc. 56(1), 73-82 (2019)

13. Kozłowska-Walania, E., Turbek, P.: Real equations for o-extremal Riemann surfaces with abelian automorphism group, preprint 
14. Macbeath, A.M.: Action of automorphisms of a compact Riemann surface on the first homology group. Bull. Lond. Math. Soc. 5, 103-108 (1973)

15. Singerman, D.: On the structure of non-Euclidean crystallographic groups. Proc. Camb. Philos. Soc. 76, 233-240 (1974)

16. Turbek, P.: Computing Equations, Automorphisms and Symmetries of Riemann Surfaces, Riemann and Klein Surfaces, Automorphisms, Symmetries and Moduli Spaces, Contemporary Mathematics, vol. 629, pp. 335-348. American Mathematical Society, Providence (2014)

Publisher's Note Springer Nature remains neutral with regard to jurisdictional claims in published maps and institutional affiliations. 